\title{
CENTROS EDUCATIVOS QUE SE AUTOEVALÚAN Y REFLEXIONAN SOBRE SUS RESULTADOS PARA MEJORAR LA ATENCIÓN A LA DIVERSIDAD
}

\section{SCHOOLS WHICH SELF-ASSESS AND REFLECT UPON THEIR RESULTS FOR IMPROVING ATTENTION TO DIVERSITY}

\author{
Pilar Arnaiz Sánchez ${ }^{1}$ \\ Miryam Martínez Rodríguez \\ Universidad de Murcia, España.
}

\section{RESUMEN}

La atención a la diversidad requiere una respuesta educativa que permita a todo el alumnado alcanzar metas acordes a sus posibilidades, lo que muchas veces no se consigue, especialmente, en educación secundaria. El objetivo de este artículo es analizar los resultados que obtienen ocho centros, cinco de educación infantil y primaria y tres de secundaria de la Región de Murcia a partir de la autoevaluación que realiza el profesorado de los logros del alumnado en la adquisición de conocimientos, valores y obtención del graduado en Secundaria. Para ello se llevó a cabo una investigación descriptiva no-experimental de carácter cuantitativo en la que participaron 130 docentes seleccionados de manera no probabilística por conveniencia. Se utilizó el instrumento ACADI (Autoevaluación de Centros para la Atención a la Diversidad desde la Inclusión), en concreto, el ámbito D "Resultados". Los análisis realizados mostraron que el profesorado tiene en cuenta la situación de partida del alumnado, especialmente del más vulnerable, y que aplica en su respuesta educativa estrategias, medidas y apoyos que facilitan el aprendizaje y la obtención del graduado en educación secundaria obligatoria. Asimismo evidencian el desarrollo de valores inclusivos, de hábitos de buena conducta y la resolución dialogada de conflictos. Se concluye afirmando que la atención a la diversidad del alumnado está presente, que la aplicación de medidas de atención a la diversidad ha mejorado el logro de los estudiantes y que hay que seguir

1 Correspondencia: Pilar Arnáiz Sánchez: Universidad de Murcia. Facultad de Educación. Departamento de Didáctica y Organización Escolar. Campus de Espinardo.30100 Murcia. Correo-e: parnaiz@um.es 
potenciando la participación de todos los agentes educativos para que la educación inclusiva impregne todas sus acciones.

Palabras clave: atención a la diversidad, educación inclusiva, calidad de la educación, progreso académico, mejora educativa.

\section{ABSTRACT}

Attention to diversity requires and educational response that enables all students to meet goals that are in line with their possibilities, something which is often not achieved, especially in secondary education. The overall aim of this article is to analyze the results obtained from eight centres - five infant and primary schools and three secondary schools in the Region of Murcia (Spain) - from self-assessments made by the teachers of students' achievements in terms of knowledge acquisition, values and gaining the high school qualification. A non experimental descriptive study of a quantitative nature was carried out with 130 teachers who, for the sake of convenience were selected no probabilistically. The ACADI (Autoevaluación de Centros para la Atención a la Diversidad desde la Inclusión - School based self-assessment of diversity awareness from an inclusive approach) was used, specifically, the field D "Results". The analyses revealed that teachers take into consideration the initial situations of their students, especially those of the most vulnerable and respond educationally by applying strategies, measures and support to facilitate learning and the certificate of graduation at the end of compulsory secondary education. Likewise, the findings show the practice of inclusive values, good behavior habits and use of dialogs to resolve conflicts. The conclusions state that attention to student diversity is present, that the application of attention to diversity measures has improved students' achievements and that there is a need to continue to boost the participation of all educational agents so that inclusive education filters through to all actions performed.

Key Words: attention to diversity, inclusive education, quality of education, academic progress, improving education.

\section{Introducción}

En los últimos años se está apostando en los centros de educación infantil, primaria y secundaria por una educación inclusiva que responda a las diversas características del alumnado, lo que requiere una continua reflexión y evaluación acerca de lo que acontece diariamente en el seno de los mismos, puesto que ello condicionará los resultados que se obtengan. La autoevaluación es necesaria si una institución quiere mejorar los resultados respecto a los procesos de enseñanza-aprendizaje que lleva a cabo, o la obtención del título de graduado de los estudiantes en la Educación Secundaria Obligatoria, entre otros (Arnaiz, De Haro, Guirao, 2015; Cantón, 2014; Villa y Thousand, 2016). Es esencial que los centros evalúen sus prácticas, que conozcan lo que están haciendo bien (fortalezas), lo que hay que cambiar (debilidades) y lo que queda por hacer (propuestas de mejora), con el fin de mejorar sus resultados en términos de calidad (Arnaiz, Azorín y García, 2015; Escarbajal, Arnaiz y Giménez, 2016; Villa, Troncoso y Díez, 2015).

San Fabián y Granda (2013) indican que toda autoevaluación debe ser eficaz y responder, desde una perspectiva democrática, a aspectos referidos al aprendizaje, desarrollo y éxito de los alumnos e implicar a todos los agentes educativos (padres y madres, profesores, alumnos, 
directivos...), siguiendo seis principios básicos: transparencia, visión global del centro, contextualización, participación, orientación a la acción y diálogo como método. De este modo, para mejorar los centros se debería potenciar el éxito de todos como respuesta equitativa al alumnado y atender a la diversidad implicada de una manera óptima. La aplicación de medidas específicas de atención a la diversidad como Adaptaciones Curriculares, Programas de Diversificación Curricular, Programas de Cualificación Profesional, algunas de ellas sustituidas en la actualidad por otras con finalidades similares, pueden constituirse en un gran apoyo para los alumnos que las precisan y contribuir a su éxito y promoción (Arnaiz, 2009; Fernández, 2014; Aramendi y Vega, 2013; Martínez, 2011).

Caminar hacia una educación inclusiva supone tener una mentalidad abierta a cambios en el seno de las instituciones, indagar acerca de sus posibilidades y aprender de sus experiencias (Ainscow, 2016; Arnaiz y Azorín, 2014; Dueñas, 2010; Florian, Black-Hawkins y Rouse, 2016; Moriña, 2008; Parrilla, 2007; Pearson, 2016). Por ello la práctica educativa ha de estar impregnada por la filosofía inclusiva cuyo ideario plantea el acceso, la presencia, la participación, la permanencia, el éxito y el logro de todos los estudiantes. En esta tarea una buena coordinación y colaboración entre profesionales promueve el cambio y facilita los procesos de mejora (Arnaiz, Escarbajal, Guirao y Martínez, 2016; Manzanares, 2013). Sin embargo, cuando se investiga acerca de lo que acontece en muchos centros y en sus aulas se encuentra que se responde al alumnado desde propuestas homogeneizadoras que no respetan sus características individuales, lo que promueve situaciones de exclusión y marginación, especialmente, en el caso de los estudiantes más vulnerables (Echeita, 2008; Escudero y Martínez, 2011; Escudero, González y Martínez, 2009). Incluso se pueden encontrar centros educativos que se consideran a sí mismos como "escuelas inclusivas" cuando verdaderamente su praxis educativa no lo es (Messiu, 2017; Muntaner, Roselló y De la Iglesia, 2016). A pesar de que es evidente la heterogeneidad del alumnado, no se observa en ellos una atmósfera adecuada donde las diferencias sean consideradas como una oportunidad para aprender y mejorar los resultados de todo el alumnado.

Montero y Silva (2012) afirman sobre este particular que, además de conseguir que todos los alumnos tengan buenos resultados, es esencial que también adquieran un alto valor personal y social para que su vida se desarrolle con el mejor bienestar posible. El Informe Delors (1996) insiste sobre este particular, exponiendo la necesidad de aplicar a la vida académica habilidades tales como "aprender a conocer", "aprender a hacer", "aprender a vivir juntos" y "aprender a ser", refiriéndose a que no solo es importante lo cognitivo (qué enseñar), sino también otros aspectos como el procedimental (cómo lo hacemos), el actitudinal (por qué o para qué) y el relacional (con quién o quiénes).

La educación inclusiva pretende conseguir la participación del alumnado y sus familias, de los docentes, de los orientadores y del resto de miembros de la comunidad educativa local donde se encuentran emplazados los centros, con el fin de crear un clima escolar que contribuya a que todos el alumnado tenga éxito, a la resolución de conflictos y a una mejor convivencia (Chenoweth, 2017; Ekins, 2017; León, 2012; Susinos, Calvo y Rojas, 2014).

En este proceso las buenas relaciones entre profesor-alumno desempeñan un papel importante en la evolución de los estudiantes y en sus actitudes hacia el aprendizaje (OCDE, 2015). Como bien muestra la investigación realizada por Berasategui y Orcasitas (2014), cuando los centros apuestan por una educación inclusiva se aprecian cambios bastante positivos a nivel personal y en las relaciones (respeto y aceptación de la diversidad, altas expectativas del profesor, aumento de la comunicación, el diálogo y las relaciones interpersonales), cambios educativos y pedagógicos (buena relación entre docente-alumno, colaboración, cooperación y clima adecuado), y la inclusión de las familias y el resto de la comunidad local en el proceso educativo.

Se trata, en definitiva, de alcanzar una educación de calidad para todos y cada uno de los alumnos, de manera que se asegure a los jóvenes la adquisición de los conocimientos, 
capacidades, destrezas y actitudes necesarias para la vida adulta (Casanova, 2012). Calidad para que toda la comunidad educativa y los agentes educativos, tanto internos como externos, estén satisfechos con los resultados que se obtienen en el centro educativo, y para adquirir una actitud de responsabilidad y compromiso que ha de ser asumida por toda la comunidad, y que se podrá conseguir si se utilizan de manera adecuada los recursos disponibles y se implica a todo y a todos (Creemers y Kyriakides, 2015; Malpica, 2013).

En consonancia con lo expresado, el presente trabajo expone un estudio cuya finalidad es conocer la autoevaluación que realiza el profesorado sobre los resultados que se obtienen en sus centros en aspectos tales como el logro de los estudiantes, los alumnos que titulan en función de las medidas específicas que se aplican, la participación y el clima de convivencia existente, entre otras, comparando si existen diferencias entre los centros de infantil y primaria, y secundaria participantes. Todo ello permitirá conocer la situación actual de los mismos y orientará sobre posibles actuaciones de mejora.

A la vista de este planteamiento el objetivo general del presente estudio se centra en analizar los resultados que obtienen los centros de educación infantil y primaria, y secundaria participantes a partir de la autoevaluación que realiza el profesorado desde una perspectiva inclusiva, para conocer las fortalezas y debilidades presentes en los mismos.

Los objetivos específicos que se desprenden de este objetivo son los siguientes:

1. Conocer el logro de los estudiantes en función de su nivel de partida, el aumento de graduados en Educación Secundaria Obligatoria y si se han aplicado para ello medidas de atención a la diversidad.

2. Examinar las medidas de atención a la diversidad implementadas por los centros para facilitar la obtención del graduado en Educación Secundaria Obligatoria del alumnado con necesidades específicas de apoyo educativo.

3. Averiguar las habilidades que se trabajan en el aula para el logro de un buen clima de centro.

4. Comprobar la actitud de respeto existente entre el alumnado ante la diversidad de características presentes en las aulas.

\section{Método}

\section{Participantes}

De una población de 10 centros educativos de educación infantil, primaria y secundaria, pertenecientes a la comarca del noroeste de la Comunidad Autónoma de la Región de Murcia, (municipios de Caravaca y Moratalla), tras un muestreo no probabilístico intencional multietápico, en la primera fase se escogieron 8 centros, 7 de ellos de titularidad pública ( 5 de infantil y primaria y 2 de secundaria) y 1 concertado que abarca las tres etapas educativas.

En la segunda fase, tal y como se muestra en la tabla 1, de una población de 346 docentes de los centros mencionados, se seleccionaron mediante un muestreo de voluntarios, 130, perteneciendo 62 de ellos a Centros de Educación Infantil y Primaria $(47,7 \%)$ y 68 a Institutos de Educación Secundaria (52,3\%). Para el análisis de datos, el centro concertado, donde se imparten las tres etapas educativas, se ha considerado como centro de educación secundaria. 
Tabla 1. Distribución muestral de docentes por centros.

\begin{tabular}{lcc}
\hline Centro educativo & Población & Muestra \\
\hline CEIP 1 & 30 & 16 \\
CEIP 2 & 12 & 9 \\
CEIP 3 & 40 & 20 \\
CEIP 4 & 46 & 14 \\
CEIP 5 & 20 & 3 \\
IES 1 & 98 & 41 \\
IES 2 & 70 & 8 \\
CEE 1 & 30 & 19 \\
\hline Total: 8 & 346 & 130 \\
\hline
\end{tabular}

\section{Variables}

Se exponen a continuación las variables que van a ser analizadas para identificar la realidad de los centros educativos que forman parte de este estudio.

- La variable predictora es la etapa educativa que ofrecen los centros. Se distinguen los centros de educación infantil y primaria de los de educación secundaria.

- Las variables criterio de este estudio se refieren a aquellos aspectos que son claves para responder adecuadamente a la diversidad de los centros y contribuyen a que los resultados que se obtengan sean fieles a la filosofía inclusiva:

- Logro del estudiante en función de su nivel de partida, obtención del título de graduado en ESO y su relación con las medidas de atención a la diversidad.

- Medidas que implementan para facilitar la titulación y la futura inserción laboral como son: Programas de Diversificación Curricular (PDC), adaptaciones curriculares significativas, Programas de Cualificación Profesional Inicial (PCPI), y relaciones con asociaciones y/o ONGs.

- Habilidades que se trabajan en el aula para la mejora del clima escolar y social del centro.

- Y actitud del alumnado ante la diversidad existente en las aulas.

\section{Instrumentos}

Para poner en marcha este estudio se ha utilizado la Guía de Autoevaluación de Centros para la Atención a la Diversidad desde la Inclusión (ACADI) que puede ser consultada en Arnaiz y Guirao (2015). Se trata de un instrumento válido y eficaz para realizar la autoevaluación institucional de centros educativos tanto de Educación Infantil y Primaria como Secundaria desde un enfoque inclusivo, que posibilita iniciar, si así se estimara necesario, procesos de cambio y mejora en los mismos.

Consta de cuatro ámbitos (contexto escolar, recursos, proceso educativo y resultados) que se dividen, a su vez, en una serie de categorías e indicadores (figura 1), que se desglosan en preguntas cerradas que se contestan a modo de escala Likert (Muy poco/ Poco/ Bastante/ Mucho). 
- Dimensión de contexto escolar: referido a la previsión y organización de acciones educativas.

- Dimensión de recursos: hace referencia a la dotación y uso de los recursos materiales e instalaciones.

- Dimensión de proceso educativo: centrado en las características que debe tener la práctica educativa, el trabajo del profesorado y en las relaciones que se establecen entre el centro y su contexto.

- Dimensión de resultados: se refiere al impacto que tienen las medidas de atención a la diversidad en las capacidades, habilidades y destrezas de los alumnos.

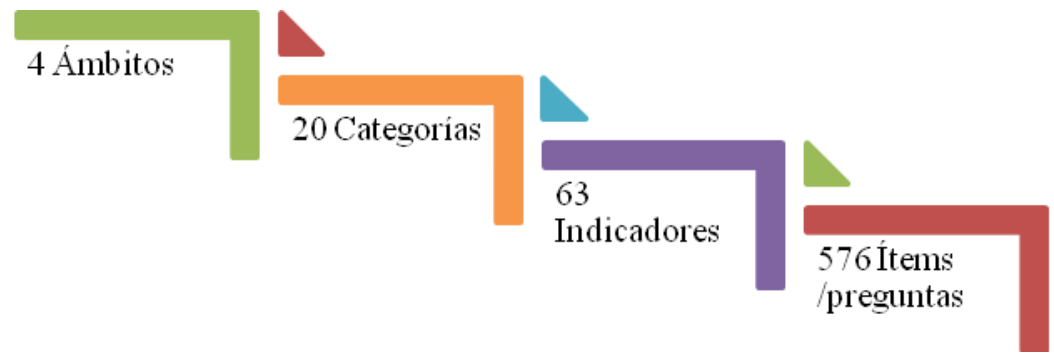

Figura 1. Estructura de ACADI

A través de los indicadores se puede valorar si la respuesta educativa que se está dando en los centros educativos para atender a la diversidad del alumnado se corresponde con el planteamiento de la educación inclusiva, si es de calidad y si contribuye a la inclusión social. De esta manera, la guía ACADI facilita los procesos de análisis de las prácticas educativas de los centros, identifica sus puntos fuertes y débiles, y posibilita, si procede, el diseño de planes de mejora, como puede comprobarse en diferentes trabajos realizados (Arnaiz, De Haro y Guirao, 2015; Arnaiz, Escarbajal, Guirao y Martínez, 2016; Arnaiz, Escarbajal y Caballero, 2017).

Los docentes deben contestar los diversos ítems en función de la realidad del centro, lo que requiere un ejercicio reflexivo que les permita detectar las fortalezas y debilidades existentes en el mismo. Dicho proceso se puede hacer por grupos de profesores o a nivel individual.

El trabajo que se presenta está centrado en el ámbito de resultados (tabla 2).

Tabla 2. Ámbito de Resultados

\begin{tabular}{llc}
\hline Categoría & Indicador & No de ítems \\
\hline $\begin{array}{l}\text { D.1 Referencia } \\
\text { a metas } \\
\text { amplias. }\end{array}$ & $\begin{array}{l}\text { D.1.1 Obtención de metas preestablecidas en el centro. Obtención de metas } \\
\text { considerando dónde comienzan los alumnos y el ritmo de su progreso. }\end{array}$ & 3 \\
\cline { 2 - 3 } & $\begin{array}{l}\text { D.1.2 Inclusión y obtención de multiplicidad de saber; saber; saber hacer, } \\
\text { saber ser y saber convivir. }\end{array}$ & 5 \\
\hline $\begin{array}{l}\text { D.2 Datos } \\
\text { objetivables de } \\
\text { rendimiento. }\end{array}$ & $\begin{array}{l}\text { D.2.1 Mejora progresiva del porcentaje de alumnos que obtiene el título de } \\
\text { graduado en E. Secundaria. }\end{array}$ & $\begin{array}{l}\text { D.2.2 Mejora progresiva del porcentaje de alumnos con medidas específicas } \\
\text { de atención a la diversidad que obtienen el título de graduado o, en su } \\
\text { defecto, se insertan adecuadamente en la vida laboral. }\end{array}$ \\
\hline $\begin{array}{l}\text { D.3 Participa- } \\
\text { ción y clima } \\
\text { del centro. }\end{array}$ & D.3.1 Mejora de las actitudes de los alumnos hacia los demás compañeros. & 8 \\
\hline
\end{tabular}




\section{Procedimiento}

En primer lugar se hizo una revisión bibliográfica para conocer la situación del tema objeto de estudio. Una vez realizada, se visitaron los centros educativos de la zona elegida con el propósito de explicarles en qué consistía la investigación y el proceso de autoevaluación que deberían llevar a cabo con el instrumento ACADI. Conocidas las posibilidades del mismo, los centros se mostraron interesados por el ámbito de resultados. Se pasaron los cuestionarios, se digitalizaron los datos mediante el programa estadístico SPSS (versión 19), se procedió a su análisis según los objetivos establecidos y se establecieron las conclusiones. Se terminó con un nuevo encuentro con los centros para devolverles la información extraída y comentarla con ellos, analizando sus fortalezas y debilidades, y la posibilidad de establecer actuaciones de mejora.

\section{Diseño y Técnicas de análisis de la información}

Este estudio tiene como finalidad explicar, controlar y predecir los fenómenos que ocurren en los centros educativos a nivel de resultados. Por consiguiente, se enmarca dentro de la investigación descriptiva no-experimental de carácter cuantitativo, puesto que la información que se obtiene para dar respuesta a los objetivos marcados se representa por medio de estadísticos (García y Martínez, 2012).

Para poder dar respuesta a los objetivos marcados en esta investigación se ha utilizado tanto la estadística descriptiva como la inferencial no paramétrica. No se han podido aplicar pruebas paramétricas puesto que las variables son de escala ordinal, la muestra es por conveniencia y la distribución de los datos no es normal (Prueba de Kolmogorov-Smirnov) y no hay homocedasticidad (Prueba de Levene). En concreto, se realizó un análisis descriptivo de los ítems del instrumento, así como la prueba de $U$ de Mann-Whitney con un nivel de significación establecido de $p<.05$. Para el análisis de los datos se utilizó el paquete estadístico SPSS (versión 19).

\section{Resultados}

La presentación de los resultados se realizará por objetivos específicos.

Objetivo 1. Conocer el logro de los estudiantes en función de su nivel de partida, el aumento de graduados en Educación Secundaria Obligatoria y si se han aplicado para ello medidas de atención a la diversidad.

Si se observa la tabla 3 , se puede afirmar que, en general, el contenido del objetivo ha sido valorado con una puntuación alta $(\mathrm{M}=3,03)$. El profesorado tiene bastante presente las características y el nivel de partida del alumnado para llevar a cabo su trabajo $(M=3,12)$, y se observa que cada vez son más los estudiantes que obtienen el graduado en ESO $(M=2,94)$. De igual forma, existe el convencimiento en el profesorado de que las medidas de atención a la diversidad que se aplican en los centros estudiados apoyan las características y necesidades personales del alumnado con dificultades, lo que propicia su evolución y titulación en la Etapa de la Educación Secundaria Obligatoria (ESO) $(M=3,05)$. 
Tabla 3. Descriptivos referidos al Objetivo 1

\begin{tabular}{|c|c|c|c|c|c|}
\hline Global Objetivo 1 & $\frac{N}{130}$ & Mín. & $\begin{array}{l}\text { Máx. } \\
4,00\end{array}$ & Media & $\begin{array}{r}\text { Desv. típ. } \\
, 521\end{array}$ \\
\hline $\begin{array}{l}\text { Se valora para cada alumno su logro en función de su nivel de } \\
\text { partida. }\end{array}$ & 130 & 2 & 4 & 3,12 & ,678 \\
\hline $\begin{array}{l}\text { En los últimos } 3 \text { años se ha producido un aumento del porcentaje de } \\
\text { alumnos que obtienen el título de Graduación ESO. }\end{array}$ & 130 & 1 & 4 & 2,94 & ,644 \\
\hline $\begin{array}{l}\text { Las medidas de atención a la diversidad desarrolladas en el centro } \\
\text { educativo han propiciado el aumento en el número de alumnos que } \\
\text { titulan. }\end{array}$ & 130 & 2 & 4 & 3,05 & ,633 \\
\hline$N$ válido & 130 & & & & \\
\hline
\end{tabular}

En la tabla 4, se muestran los mismos estadísticos descriptivos, segmentado la información por etapas educativas.

Tabla 4. Descriptivos referidos al Objetivo 1, en función de la etapa educativa

\begin{tabular}{|c|c|c|c|c|c|c|c|c|c|c|}
\hline & \multicolumn{6}{|c|}{$\begin{array}{c}\text { Centros de Educación Infantil } \\
\text { y Primaria }\end{array}$} & \multicolumn{4}{|c|}{$\begin{array}{l}\text { Centros de Educación } \\
\text { Secundaria }\end{array}$} \\
\hline & $N$ & Mín. & Máx. & Media & Desv. típ. & $N$ & Mín. & Máx. & Media & Desv. típ. \\
\hline Global Objetivo 1 & 62 & 2,00 & 4,00 & 3,21 & 422 & 68 & 1,67 & 4,00 & 2,87 & .550 \\
\hline $\begin{array}{l}\text { Se valora para cada alumno su } \\
\text { logro en función de su nivel de } \\
\text { partida. }\end{array}$ & 62 & 2 & 4 & 3,37 & ,633 & 68 & 2 & 4 & 2,88 & ,636 \\
\hline $\begin{array}{l}\text { En los últimos } 3 \text { años se ha } \\
\text { producido un aumento del } \\
\text { porcentaje de alumnos que } \\
\text { obtienen el título de Graduación } \\
\text { ESO. }\end{array}$ & 62 & 1 & 4 & 3,13 & ,495 & 68 & 1 & 4 & 2,76 & ,715 \\
\hline $\begin{array}{l}\text { Las medidas de atención a la } \\
\text { diversidad desarrolladas en el } \\
\text { centro educativo han propiciado } \\
\text { el aumento en el número de } \\
\text { alumnos que titulan. }\end{array}$ & 62 & 2 & 4 & 3,15 & ,596 & 68 & 2 & 4 & 2,96 & ,656 \\
\hline$N$ válido & 62 & & & & & 68 & & & & \\
\hline
\end{tabular}

Como muestra la tabla 4, el profesorado de los centros de educación infantil y primaria valora bastante alto la necesidad de tener en cuenta los conocimientos previos del alumnado en el proceso de enseñanza-aprendizaje, así como la importancia de aplicar medidas de atención a la diversidad que apoyen al alumnado más vulnerable para que obtenga el graduado en ESO $(\mathrm{M}=3,21)$. En cambio el profesorado de los centros de educación secundaria valora todo ello más bajo $(M=2,87)$ de forma general. En función de la etapa educativa, la prueba de Mann-Whitney $(U=1256,0)$ pone de manifiesto la existencia de diferencias significativas $(P=, 000)$, a favor de educación infantil y de primaria.

Objetivo 2. Examinar las medidas de atención a la diversidad implementadas por los centros para facilitar la obtención del graduado en Educación Secundaria Obligatoria del alumnado con necesidades específicas de apoyo educativo.

Como se puede comprobar en la tabla 5 , las valoraciones del profesorado, respecto de este objetivo han sido positivas ( $M=2,81)$. Los docentes afirman que en sus centros han aumentado notablemente $(\mathrm{M}=3,02)$ al final de la ESO las competencias cívicas y ciudadanas en los 
estudiantes con necesidades específicas de apoyo educativo gracias a la aplicación de las Adaptaciones Curriculares. Asimismo, indican que los Programas de Diversificación Curricular $(\mathrm{M}=2,84)$ han ayudado a estos alumnos a finalizar con éxito sus estudios y obtener el graduado, mientras que los Programas de Cualificación Profesional Inicial $(M=2,78)$ han mejorado su tránsito hacia la vida laboral. El profesorado afirma en cambio que el trabajo conjunto con otros agentes educativos como asociaciones y ONGs no ha sido tan estrecho como se esperaba $(M=2,58)$.

Tabla 5. Descriptivos referidos al Objetivo 2

\begin{tabular}{|c|c|c|c|c|c|}
\hline Global Objetivo 2 & $\frac{N}{130}$ & Mín. & Máx. & \begin{tabular}{c|c} 
Media \\
2,81
\end{tabular} & $\frac{\text { Desv. típ. }}{585}$ \\
\hline $\begin{array}{l}\text { Existe un porcentaje elevado de alumnos que obtienen el título de } \\
\text { Graduado en ESO a través de un programa de Diversificación Curricular. }\end{array}$ & 130 & 1 & 4 & 2,84 & ,755 \\
\hline $\begin{array}{l}\text { Los alumnos con adaptaciones curriculares significativas adquieren, al } \\
\text { finalizar la ESO, competencia social y ciudadana así como competencia } \\
\text { en autonomía e iniciativa personal. }\end{array}$ & 130 & 1 & 4 & 3,02 & 659 \\
\hline $\begin{array}{l}\text { Existen relaciones con asociaciones y/o ONGs para planificar el tránsito } \\
\text { de los alumnos a la vida activa. }\end{array}$ & 130 & 1 & 4 & 2,58 & ,913 \\
\hline $\begin{array}{l}\text { El porcentaje de alumnos que cursan y finalizan con éxito los Programas } \\
\text { de Cualificación Profesional Inicial que se desarrollan en el centro ha } \\
\text { aumentado. }\end{array}$ & 130 & 1 & 4 & 2,78 & ,838 \\
\hline$N$ válido & 130 & & & & \\
\hline
\end{tabular}

En la siguiente tabla (ver tabla 6) se muestra el mismo estudio descriptivo segmentando la información por etapas educativas. Se puede comprobar que en los centros educativos de educación infantil y primaria se valora de forma más positiva los resultados que se obtienen $(M=3,12)$ que en los centros de secundaria $(M=2,53)$. En estos, de manera frecuente, los alumnos con necesidades específicas de apoyo educativo consiguen el graduado $(\mathrm{M}=2,74)$, adquieren competencias sociales $(\mathrm{M}=2.76)$. Pero se valora con una puntuación más baja el hecho de que el título se obtenga a través de programas de Diversificación Curricular $(M=2,59)$ y que se preparen para el mundo laboral con los Programas de Cualificación Profesional Inicial $(M=2,35)$. Asimismo, el trabajo conjunto con las asociaciones y ONGs lo señalan como insuficiente $(M=2.21)$.

Tabla 6. Descriptivos referidos al Objetivo 2, en función de la etapa educativa

\begin{tabular}{|c|c|c|c|c|c|c|c|c|c|c|}
\hline \multirow{3}{*}{ Global Objetivo 2} & \multicolumn{5}{|c|}{$\begin{array}{c}\text { Centros de Educación Infantil y } \\
\text { Primaria }\end{array}$} & \multicolumn{5}{|c|}{ Centros de Educación Secundaria } \\
\hline & $\mathrm{N}$ & Mín. & Máx. & Media & Desv. típ. & $N$ & Mín. & Máx. & Media & Desv. típ. \\
\hline & 62 & 2,00 & 4,00 & 3,12 &, 501 & 68 & 1,60 & 4,00 & 2,53 & 514 \\
\hline $\begin{array}{l}\text { Existe un porcentaje elevado de } \\
\text { alumnos que obtienen el título de } \\
\text { Graduado en ESO a través de un } \\
\text { programa de Diversificación } \\
\text { Curricular. }\end{array}$ & 62 & 2 & 4 & 3,11 & 655 & 68 & 1 & 4 & 2,59 & ,758 \\
\hline $\begin{array}{l}\text { Los alumnos con adaptaciones } \\
\text { curriculares significativas adquieren, } \\
\text { al finalizar la ESO, competencia } \\
\text { social y ciudadana así como } \\
\text { competencia en autonomía e } \\
\text { iniciativa personal. }\end{array}$ & 62 & 2 & 4 & 3,29 & 611 & 68 & 1 & 4 & 2,76 & ,601 \\
\hline $\begin{array}{l}\text { Existen relaciones con asociaciones } \\
\text { y/o ONGs para planificar el tránsito } \\
\text { de los alumnos a la vida activa. }\end{array}$ & 62 & 1 & 4 & 3,00 & 810 & 68 & 1 & 4 & 2,21 & ,839 \\
\hline
\end{tabular}




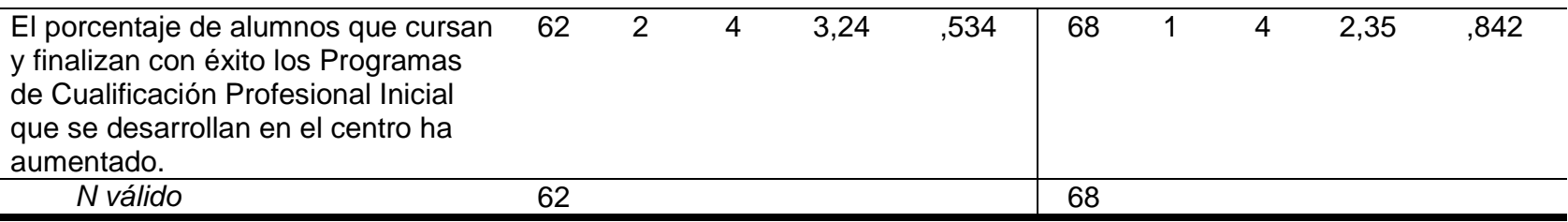

En función de la etapa educativa, la prueba de Mann-Whitney $(U=853,5)$ pone de manifiesto la existencia de diferencias significativas $(P=, 000)$, a favor de educación infantil y de primaria.

Objetivo 3. Averiguar las habilidades que se trabajan en el aula para el logro de un buen clima de centro.

En la tabla 7 se puede comprobar que la mayoría de los docentes, de manera frecuente, pone el acento en el logro de habilidades relacionadas con un buen clima de centro $(M=3,32)$. La mayoría del profesorado indica que promueve valores que proporcionen una buena convivencia entre el alumnado $(M=3,38)$, el desarrollo de hábitos para adquirir una buena conducta $(M=3,37)$, habilidades para dialogar ante los conflictos $(M=3,34)$ y hábitos de buena conducta $(M=3,18)$.

Tabla 7. Descriptivos referidos al Objetivo 3

\begin{tabular}{lccccr}
\hline \multicolumn{1}{c}{ Global Objetivo 2 } & $N$ & Mínimo & Máximo & Media & Desv. típ. \\
\cline { 2 - 6 } & 130 & 1,25 & 4,00 & 3,32 &, 575 \\
\hline $\begin{array}{l}\text { La educación del alumno se centra el aprendizaje } \\
\text { de hábitos para desarrollar su vida con el mayor } \\
\text { bienestar posible. }\end{array}$ & 130 & 1 & 4 & 3,18 &, 698 \\
$\begin{array}{l}\text { Se promueve la adquisición de valores de } \\
\text { convivencia. }\end{array}$ & 130 & 1 & 4 & 3,38 &, 696 \\
$\begin{array}{l}\text { Se facilita el aprendizaje para la resolución } \\
\text { dialogada de conflictos. }\end{array}$ & 130 & 2 & 4 & 3,34 &, 629 \\
$\begin{array}{l}\text { Se trabaja específicamente sobre el aprendizaje } \\
\text { de hábitos de buena conducta. }\end{array}$ & 130 & 1 & 4 & 3,37 &, 706 \\
\hline$\quad$ válido & 130 & & & & \\
\hline
\end{tabular}

A continuación, se presentan los mismos estadísticos descriptivos según la etapa educativa que ofrecen los centros (ver tabla 8). El profesorado de educación infantil y primaria, a nivel muestral, da una valoración más alta a las habilidades relacionadas con el buen comportamiento de sus alumnos $(M=3,61)$, a los valores para alcanzar un buen clima de centro $(M=3,60)$, solucionar los posibles problemas $(\mathrm{M}=3,48)$ y desarrollar habilidades que aumenten el bienestar del alumnado $(M=3,44)$. En cambio, los docentes de secundaria obtienen unos valores un poco más bajos tanto a nivel global, como en cada una de los ítems indicados. 
Tabla 8. Descriptivos referidos al Objetivo 3, en función de la etapa educativa

\begin{tabular}{|c|c|c|c|c|c|c|c|c|c|c|}
\hline \multirow{3}{*}{ Global Objetivo 3} & \multicolumn{5}{|c|}{$\begin{array}{c}\text { Centros de Educación Infantil } \\
\text { y Primaria }\end{array}$} & \multicolumn{5}{|c|}{ Centros de Educación Secundaria } \\
\hline & $\mathrm{N}$ & Mín. & Máx. & Media & Desv. típ. & $N$ & Mín. & Máx. & Media & Desv. típ. \\
\hline & 62 & 2,25 & 4,00 & 3,53 &, 426 & 68 & 1,25 & 4,00 & 3,12 &, 624 \\
\hline $\begin{array}{l}\text { La educación del alumno se } \\
\text { centra el aprendizaje de hábitos } \\
\text { para desarrollar su vida con el } \\
\text { mayor bienestar posible. }\end{array}$ & 62 & 2 & 4 & 3,44 &, 562 & 68 & 1 & 4 & 2,94 & ,731 \\
\hline $\begin{array}{l}\text { Se promueve la adquisición de } \\
\text { valores de convivencia. }\end{array}$ & 62 & 2 & 4 & 3,60 & ,557 & 68 & 1 & 4 & 3,18 & ,752 \\
\hline $\begin{array}{l}\text { Se facilita el aprendizaje para la } \\
\text { resolución dialogada de } \\
\text { conflictos. }\end{array}$ & 62 & 2 & 4 & 3,48 & ,565 & 68 & 2 & 4 & 3,21 & ,659 \\
\hline $\begin{array}{l}\text { Se trabaja específicamente } \\
\text { sobre el aprendizaje de hábitos } \\
\text { de buena conducta. }\end{array}$ & 62 & 2 & 4 & 3,61 &, 523 & 68 & 1 & 4 & 3,15 & ,778 \\
\hline$N$ válido & 62 & & & & & 68 & & & & \\
\hline
\end{tabular}

Finalmente, en función de la etapa educativa, la prueba de Mann-Whitney $(U=1277,5)$ pone de manifiesto la existencia de diferencias significativas $(P=, 000)$, a favor de educación infantil y de primaria.

Objetivo 4. Comprobar la actitud de respeto existente entre el alumnado ante la diversidad características presentes en las aulas.

En la tabla 9 se presenta la valoración del profesorado respecto a los contenidos que conforman este objetivo que, como se puede observar ha obtenido una puntuación bastante alta $(M=3,31)$. Ello obedece a que se promueven constantemente actitudes de respeto $(M=3,37)$ y, en el caso de que se produzcan conflictos entre iguales, el docente siempre desarrolla un rol de mediador $(\mathrm{M}=3,57)$ para su resolución. Asimismo, el profesorado tiente bastante presente la voz del alumnado en los temas que les concierne, debatiendo, por ejemplo, las normas de aula $(M=3,25)$, las cuales son bastante claras $(M=3,55)$, y escuchando las propuestas de los estudiantes para mejorar así el clima escolar $(M=3,13)$. En el caso de que se produzcan situaciones graves se conocen bastante bien las estrategias que han de ponerse en marcha $(M=3,27)$, previniendo y reduciendo el posible "bullying" entre los estudiantes $(M=3,05)$.

Tabla 9. Descriptivos referidos al objetivo 4

\begin{tabular}{lccccc}
\hline \multicolumn{1}{c}{ Global Objetivo 4 } & $N$ & Mín. & Máx. & Media & Desv. típ. \\
\cline { 2 - 6 } & 130 & 1,43 & 4,00 & 3,31 &, 479 \\
\hline $\begin{array}{l}\text { Las faltas de respeto o la utilización de actitudes negativas hacia } \\
\text { los compañeros son tratadas en clase y existen los mecanismos } \\
\text { y momentos para ello. }\end{array}$ & 130 & 1 & 4 & 3,37 &, 637 \\
\hline $\begin{array}{l}\text { El profesor actúa como mediador entre los compañeros causa del } \\
\text { conflicto. }\end{array}$ & 130 & 2 & 4 & 3,57 &, 542 \\
\hline $\begin{array}{l}\text { Las normas del comportamiento del aula son consistentes y } \\
\text { explícitas. }\end{array}$ & 130 & 2 & 4 & 3,55 &, 558 \\
\hline $\begin{array}{l}\text { Se debate y se consensúa con el alumnado las normas de } \\
\text { comportamiento del aula. }\end{array}$ & 130 & 1 & 4 & 3,25 &, 771 \\
\hline $\begin{array}{l}\text { Se consulta a los alumnos cómo se pueden mejorar el clima } \\
\text { social del aula. }\end{array}$ & 130 & 1 & 4 & 3,13 &, 709 \\
\hline
\end{tabular}




\begin{tabular}{llllll}
\hline $\begin{array}{l}\text { Hay procedimientos claros, entendidos por los alumnos y los } \\
\text { docentes para responder a los comportamientos extremos. }\end{array}$ & 130 & 1 & 4 & 3,27 &, 691 \\
$\begin{array}{l}\text { Se han reducido las relaciones de abuso de poder entre iguales o } \\
\text { "bullying". }\end{array}$ & 130 & 1 & 4 & 3,05 &, 856 \\
\hline$N$ válido & 130 & & & \\
\hline
\end{tabular}

En la tabla 10 se presentan los datos anteriores diferenciados por etapas educativas, observándose que las diferencias en función de la etapa educativa entre los centros de educación infantil y primaria $(M=3,43)$, y secundaria $(M=3,21)$ son pocas. Se puntúa de una manera más alta en los centros de educación infantil y primaria el fomento de actitudes positivas para evitar los posibles conflictos $(M=3,56)$, la colaboración de todos para la elaboración de las normas $(M=3,47)$ y su construcción de forma sólida y clara $(\mathrm{M}=3,69)$. Asimismo, el docente está bastante presente en posibles conflictos con un perfil de mediador $(M=3,71)$ y, en el caso de situaciones más graves, todos conocen y entienden bastante bien el procedimiento a seguir $(M=3,34)$. Destaca que en los centros se han ido reduciendo las situaciones de abuso de poder entre iguales tanto en secundaria $(M=3,10)$ como en educación infantil y primaria $(M=3,00)$.

Tabla 10. Descriptivos referidos al Objetivo 4, en función de la etapa educativa

\begin{tabular}{|c|c|c|c|c|c|c|c|c|c|c|}
\hline \multirow{3}{*}{ Global Objetivo 4} & \multicolumn{5}{|c|}{$\begin{array}{c}\text { Centros de Educación Infantil } \\
\text { y Primaria }\end{array}$} & \multicolumn{5}{|c|}{ Centros de Secundaria } \\
\hline & $N$ & Mín. & Máx. & Media & $\begin{array}{l}\text { Desv. } \\
\text { típ. }\end{array}$ & $N$ & Mín. & Máx. & Media & $\begin{array}{l}\text { Desv. } \\
\text { típ. }\end{array}$ \\
\hline & 62 & 2,86 & 4,00 & 3,43 & ,363 & 68 & 1,43 & 4,00 & 3,21 &, 54426 \\
\hline $\begin{array}{l}\text { Las faltas de respeto o la utilización } \\
\text { de actitudes negativas hacia los } \\
\text { compañeros son tratadas en clase y } \\
\text { existen los mecanismos y momentos } \\
\text { para ello. }\end{array}$ & 62 & 2 & 4 & 3,56 &, 532 & 68 & 1 & 4 & 3,19 & 675 \\
\hline $\begin{array}{l}\text { El profesor actúa como mediador } \\
\text { entre los compañeros causa del } \\
\text { conflicto. }\end{array}$ & 62 & 3 & 4 & 3,71 & ,458 & 68 & 2 & 4 & 3,44 & ,583 \\
\hline $\begin{array}{l}\text { Las normas del comportamiento del } \\
\text { aula son consistentes y explícitas. }\end{array}$ & 62 & 3 & 4 & 3,69 & ,465 & 68 & 2 & 4 & 3,41 & ,604 \\
\hline $\begin{array}{l}\text { Se debate y se consensúa con el } \\
\text { alumnado las normas de } \\
\text { comportamiento del aula. }\end{array}$ & 62 & 2 & 4 & 3,47 &, 535 & 68 & 1 & 4 & 3,06 & ,896 \\
\hline $\begin{array}{l}\text { Se consulta a los alumnos cómo se } \\
\text { pueden mejorar el clima social del } \\
\text { aula. }\end{array}$ & 62 & 2 & 4 & 3,27 & ,577 & 68 & 1 & 4 & 3,00 & ,792 \\
\hline $\begin{array}{l}\text { Hay procedimientos claros, } \\
\text { entendidos por los alumnos y los } \\
\text { docentes para responder a los } \\
\text { comportamientos extremos. }\end{array}$ & 62 & 2 & 4 & 3,34 & ,626 & 68 & 1 & 4 & 3,21 & ,744 \\
\hline $\begin{array}{l}\text { Se han reducido las relaciones de } \\
\text { abuso de poder entre iguales o } \\
\text { "bullying". }\end{array}$ & 62 & 1 & 4 & 3,00 & ,887 & 68 & 1 & 4 & 3,10 & ,831 \\
\hline$N$ válido & 62 & & & & & 68 & & & & \\
\hline
\end{tabular}

En función de la etapa educativa, la prueba de Mann-Whitney $(U=1579,5)$ pone de manifiesto la existencia de diferencias significativas $(P=, 013)$, a favor de educación infantil y de primaria. 


\section{Conclusiones}

En términos generales cabe indicar que la autoevaluación realizada por los docentes ha permitido conocer la percepción del profesorado en cuanto a los resultados que están obteniendo sus centros respecto de la atención a la diversidad del alumnado en una escuela inclusiva (Arnáiz, Escarbajal y Caballero, 2017; San Fabián y Granda, 2013; Villa y Thousand, 2016). En términos generales se ha constatado que las valoraciones vertidas por los centros de educación infantil y primaria son más positivas que las de los centros de secundaria. Quizás las causas residan en la mayor exigencia académica de la educación secundaria y en el cambio que se produce en esta etapa en el desarrollo de los procesos de enseñanza-aprendizaje, todo lo cual produce mayores dificultades para enseñar y aprender, y seguir el ritmo académico de las clases en el caso del alumnado más vulnerable (Casanova, 2012; Escudero, González y Martínez, 2009; Fernández, 2014; Manota y Melendro, 2016; Portela y Nieto, 2015).

Los ocho centros son conscientes de la necesidad de atender a la diversidad del alumnado desde los planteamientos de la educación inclusiva, considerando que para ello es primordial tener en cuenta las características y las necesidades de los estudiantes (Florian, Black-Hawkins y Rouse, 2016; Moriña, 2008, Messiou, 2017; Pearson, 2016). Por ello, el profesorado se plantea el éxito de todos y cada uno de alumnos proporcionándoles una educación personalizada que parta de sus conocimientos previos y les permita ver cómo mejoran sus logros académicos. Al mismo tiempo la mayoría de los participantes señala que son escasas las relaciones con asociaciones y ONGs, aunque tienen claro que es muy recomendable la inclusión de este tipo de agentes en los centros ya que sus actuaciones contribuyen al desarrollo integral de los alumnos (Berasategui y Orcasitas, 2014; Parrilla, 2007).

La inclusión y la participación de todos los agentes en el proceso educativo del alumnado contribuyen a la creación de un clima de centro adecuado y a reducir poco a poco el fracaso escolar (Antelm Lanzat, Gil-López, Cacheiro-González, 2015; Susinos, Calvo y Rojas, 2014; González, García, Ruiz y Muñoz, 2015). Cabe destacar la relación que establece el profesorado entre la aplicación de las medidas específicas de atención a la diversidad con el aumento de alumnos con necesidades de apoyo educativo que obtienen el título de la ESO. Esto es importante ya que corrobora que cuando estas medidas se aplican para apoyar la inclusión y el éxito académico de los estudiantes son de gran utilidad y producen satisfacción en los docentes (Arnáiz, 2009; Martínez, 2011). No obstante, hemos encontrado algunas reservas en el profesorado de secundaria que manifiesta cierto desagrado ante la obtención de este título tras la aplicación de medidas específicas, parece como si se devaluara por ello. Esta idea contrasta con la filosofía de la inclusión educativa que defiende los principios de equidad, igualdad y justicia, especialmente, en el caso del alumnado más vulnerable que requiere en ocasiones la aplicación de apoyos tales como Adaptaciones Curriculares, Programas de Diversificación Curricular y de Cualificación Profesional Inicial para poder aprender y progresar.

En general los docentes destacan la necesidad de desarrollar toda una serie de capacidades, habilidades y destrezas que favorezca experiencias de vida positivas para lograr el mayor bienestar posible, promoviendo valores de convivencia, facilitando la resolución dialogada de conflictos y trabajando hábitos de buena conducta (Delors, 1996; Susinos y Rodríguez-Hoyos, 2011). Las mismas se consideran fundamentales para el alumnado y deben adquirirse a lo largo de la vida para que puedan integrarse en la sociedad de la forma más positiva, dado su alto valor personal y social (Montero y Silva, 2012). La mayoría de los docentes opinan que las trabajan con bastante frecuencia, aunque pueden ser mejoradas poniendo en práctica programas y actividades para la convivencia (Palomares, 2008), sobre todo, aquellos que promueven hábitos para un 
mayor bienestar y para una resolución dialogada de conflictos. De igual forma, todas estas habilidades son más visibles en los centros de educación primaria que en los de secundaria.

La actitud de respeto entre el alumnado ante la diversidad de características en las aulas es bastante buena en los centros estudiados. Los docentes tratan los problemas en el aula utilizando estrategias que alertan ante el abuso entre iguales (Cerezo, Sánchez, Ruiz y Arense, 2015). Del mismo modo, para favorecer un buen clima escolar y social se consulta, debate y consensúa con el alumnado las normas de comportamiento, escuchando su voz. De esta forma, se tienen en cuenta las recomendaciones de Fielding (2011) cuando indica que no basta con escuchar a los alumnos, sino que sus opiniones e ideas han de ser tenidas en cuenta, ya que ello favorece las buenas relaciones entre profesor-alumno y mejora las actitudes de los estudiantes hacia el aprendizaje. Que los centros consulten e incluyan la participación del alumnado contribuye a la mejora escolar y a conocer las demandas principales de éstos en términos de asistencia y actitud de los estudiantes (Susinos y Rodríguez- Hoyos, 2011).

Cabe indicar, para finalizar, que la filosofía inclusiva está cada vez más presente en los centros analizados, no obstante, deben seguir trabajando para que impregne todas sus acciones. Destaca como fortaleza que los centros valoren el logro de los estudiantes en función de su nivel de partida, lo que puede ser mejorado, principalmente, en educación secundaria. El desarrollo de medidas de atención a la diversidad también ha dado lugar al crecimiento de los alumnos que titulan (PDC) o en su defecto les ha facilitado una futura inserción laboral (PCPI). La participación de todos los agentes en el proceso educativo del alumnado, la organización, gestión y coordinación democrática, el análisis de las barreras que impiden la asistencia al centro, la recopilación de información sobre las ausencias registradas, entre otras, hay que potenciarlas puesto que favorecen la creación de un clima de centro adecuado, reduciendo el fracaso escolar, especialmente, en los centros de secundaria (Fernández, 2014).

Asimismo, se debe apostar por una mayor relación y colaboración con asociaciones y ONGs para ayudar a planificar el tránsito del alumnado a la vida activa. Aunque la participación de los agentes educativos en los centros cada vez es mayor, encontrándose diferencias a favor de los centros de infantil y primaria, debe ser mejorada. Según Rodríguez Navarro (2015), la participación informativa, consultiva, decisoria, evaluativa y educativa de las familias y otros agentes educativos en los centros es esencial ya que supone un gran enriquecimiento y mejora del clima escolar. Por ello, hay que seguir incidiendo en estos aspectos en los centros analizados, sobre todo, en los de educación secundaria (Ainscow y West, 2008), teniendo el departamento de Orientación un papel protagonista en ello.

Los docentes hacen hincapié en el desarrollo de toda una serie de capacidades, habilidades y destrezas relacionadas con el "saber ser" y el "saber convivir", lo que favorece en los estudiantes experiencias de vida positivas para lograr el mayor bienestar posible, el desarrollo de valores de convivencia intercultural, de hábitos de buena conducta y la resolución dialogada de conflictos en el alumnado. Aun así, estos resultados pueden ser mejorados, de manera particular, en los centros de educación secundaria en aspectos tales como el desarrollo de experiencias de vida para el mayor bienestar posible, la resolución dialogada de conflictos y un mejor aprendizaje.

Somos conscientes de que este estudio tiene sus limitaciones derivadas de su realización en una sola comarca de la Región de Murcia, la muestra ha sido reducida, lo que ha limitado tanto los análisis que se han podido realizar como la posibilidad de generalizar los hallazgos encontrados y establecidos en las conclusiones. Seguiremos investigando en esta línea, tratando de ampliar la muestra y estudiando otras variables con el fin de seguir contribuyendo al desarrollo de centros educativos cada vez más inclusivos y atentos a la diversidad. 


\section{Referencias bibliográficas}

Ainscow, M. (2016). Equidad, el gran reto de los sistemas educativos. En H.J. Malone (Ed.), El rumbo de la transformación educativa. Temas, retos globales y lecciones sobre la reforma estructural (101-106). México: Fondo de Cultura Económica.

Ainscow, M. y West, M. (2008). Mejorar las escuelas urbanas. Madrid: Narcea.

Antelm Lanzat, A. M.; Gil-López, A. y Cacheiro-González, M. L. (2015). Análisis del fracaso escolar desde la perspectiva del alumnado y su relación con el estilo de aprendizaje. Educación y Educadores, 18(3), 471-489.

Aramendi, P. y Vega, A. (2013). Los Programas de Cualificación Profesional. La perspectiva del alumnado del País Vasco. Revista de Educación, 360, 436-460.

Arnaiz, P. (2009). Análisis de las medidas de atención a la diversidad en la Educación Secundaria Obligatoria. Revista de Educación, 349, 203-223.

Arnaiz, P. y Azorin, C.M. (2014). Autoevaluación docente para la mejora de los procesos educativos en escuelas que caminan hacia la inclusión. Revista Colombiana de Educación, 67, 227-245.

Arnaiz, P.; Azorin, C.M. y García-Sanz, M.P. (2015). Evaluación de planes de mejora en centros educativos de orientación inclusiva. Profesorado. Revista de currículum y formación del profesorado, 19(3), 326-346.

Arnaiz, P.; De Haro, R. y Guirao, J.M. (2015). La evaluación en educación primaria como punto de partida para el desarrollo de planes de mejora inclusivos en la Región de Murcia. Revista Electrónica Interuniversitaria de Formación del Profesorado, 18, 103-122.

Arnaiz, P.; Escarbajal, A.; Guirao, J.M. y Martínez, R. (2016). Building inclusive processes for school improvement: a case study. Journal of Research in Special Education Needs, 16(1), 290-294.

Arnaiz, P. y Guirao, J.M. (2015). La autoevaluación de centros en España para la atención a la diversidad desde una perspectiva inclusiva. ACADI. Revista Electrónica Interuniversitaria de Formación del Profesorado, 18, 45-101.

Arnaiz, P.; Escarbajal, A. y Caballero, C.M. (2017). El impacto del contexto escolar en la inclusión educativa. Revista Educación Inclusiva, 10(2), 195-210.

Berasategui, N. y Orcasitas, J. R. (2014). Buenas prácticas educativas a través de las voces de las familias y el alumnado con EM. Revista de Educación Inclusiva, 7, 68-83.

Cantón, I. (2014). Mejora y calidad en los centros educativos. Modelos y propuestas. En I. Cantón y M. Pino (Eds.), Organización de centros educativos en la sociedad del conocimiento (251-272). Madrid: Alianza Editorial.

Casanova, M. A (2012). El diseño curricular como factor de calidad educativa. Revista Electrónica Iberoamericana sobre Calidad, Eficacia y Cambio en Educación, 10, 6-20.

Cerezo, F.; Sánchez, C.; Ruiz, C. y Arense, J.J. (2015). Roles en bullying de adolescentes y preadolescentes, y su relación con el clima social y los estilos educativos parentales. Revista de Psicodidáctica, 20(1), 139-155.

Creemers, B. y Kyriakides, L. (2015). Developing, testing and using theoretical models of educational effectiveness for promoting quality in education. School Effectiveness and School Improvement, 26(1), 102-119. 
Chenoweth, K. (2017). Schools that succeed. How Educators Marshal the Power of Systems for Improvement. Massachusetts: Harvard Education Press.

Delors, J. (coord.) (1996). La Educación encierra un tesoro. Madrid: Santillana.

Dueñas, M.L. (2010). Educación inclusiva. Revista Española de Orientación y Psicopedagogía, 21(2), 358-366.

Echeita, G. (2008). Inclusión y exclusión educativa. "Voz y quebranto". Revista Electrónica Iberoamericana sobre Calidad, Eficacia y Cambio en Educación, 6(2), 9-18.

Ekins, A. (2017). Reconsidering inclusion. Sustaining and building inclusive practices in schools. London: Routledge.

Escarbajal, A.; Arnaiz, P. y Gualdo, A. (2017). Evaluación de las fortalezas y debilidades del proceso educativo en centros de infantil, primaria y secundaria desde una perspectiva inclusiva. Revista Complutense de Educación, 28(2), 427-443.

Escudero, J. M.; González, Mํ‥T. y Martínez, B. (2009). El fracaso escolar como exclusión educativa: comprensión, políticas y prácticas. Revista Iberoamericana de Educación, 50, 4164.

Escudero, J.M. y Martínez, B. (2011). Educación inclusiva y cambio escolar. Revista Iberoamericana de Educación, 55, 85-105.

Fernández, J.M. (2014). Prácticas educativas de orientación inclusiva ante el fracaso escolar. Estudio de caso. Journal for Educators, Teachers and Trainers, 5(2), 235-246.

Fielding, M. (2011). La voz del alumnado y la inclusión educativa: una aproximación democrática radical para el aprendizaje intergeneracional. Revista Interuniversitaria de Formación del Profesorado, 70, 31-62.

Florian, L.: Black-Hawkins, K. y Rouse, M. (2016). Achievement and inclusion in schools. London: Routledge.

García M. P. y Martínez, P. (2012). Guía práctica para la realización de Trabajos Fin de Grado y Trabajos Fin de Máster. Murcia: Editum.

González, S; García, M.P.; Ruiz, F. y Muñoz, J.M. (2015). Factores de riesgo del abandono escolar desde la perspectiva del profesorado de Educación Superior Obligatoria en Andalucía (España). Profesorado: Revista de curriculum y formación del profesorado, 19(3), 226-245.

León, M.J. (2012). El liderazgo para y en la escuela. Educatio Siglo XXI, 30(1), 133-160.

Malpica, F. (2013). Calidad De La Práctica Educativa. Referentes, Indicadores y Condiciones para mejorar la enseñanza aprendizaje. Barcelona: Graó.

Manota, M.A. y Melendro, M. (2016). Clima de aula y buenas prácticas docentes con adolescentes vulnerables: más allá de los contenidos académicos. Contextos Educativos, 19, 55-74.

Manzanares, A. (2013). Sistemas integrados de orientación. Una propuesta para la articulación de estructuras y servicios a nivel local. Revista Española de Orientación y Psicopedagogía, 24(1), 62-77.

Martínez, B. (2011). Medidas de atención a la diversidad y su papel en la prevención del fracaso escolar. Organización y Gestión Educativa, 19(5), 20-23.

Messiou, K. (2017). Research in the field of inclusive education: time for a rethink? International Journal of Inclusive Education, 21(2), 146-159.

Montero, A. J. G. y Silva, M. J. F. (2012). Competencias básicas: Un marco legislativo, teórico y aplicativo. INFAD. Revista de Psicología, 2, 59-68. 
Moriña, A. (2008). ¿Cómo hacer que un centro educativo sea inclusivo? Análisis del diseño, desarrollo y resultados de un programa formativo. Revista de Investigación Educativa, 26(2), 521-538.

Muntaner, J.J.; Roselló, M. R. y De La Iglesia, B. (2016). Buenas prácticas en educación inclusiva. Revista Educatio Siglo XXI, 34(1), 31-50.

OCDE (2015). Do teacher-student relations affect students' well-being at school? PISA in Focus, $50,1-4$.

Parrilla, M. A. (2007). El desarrollo local e institucional de proyectos educativos inclusivos. Perspectiva CEP, 14, 17-31.

Palomares, A. (2008). Contextos educativos. Revista Educación, 11, 71-94.

Pearson, S. (2016). Rethinking Children and Inclusive Education. Opportunities and Complexities. London: Bloomsbury.

Portela, A. y Nieto, J.M. (2015). Alumnos y profesores: perfiles y características en una escuela de vulnerabilidad educativa. En M.T. González (coord.), La vulnerabilidad escolar y los programas de Cualificación Profesional Inicial: apuntes para la formación profesional (61-113). Madrid: Wolters Kluwer.

Rodríguez, H. (2015). Marco de referencia internacional y modelos emergentes en educación inclusiva. Análisis sobre el enfoque inclusivo en educación. En A. Escarbajal Frutos. (Eds.), Comunidades interculturales y democráticas (93-51). Madrid: Narcea.

San Fabián, J.L. y Granda, A. (2013). Principios básicos de autoevaluación. En J.L. San Fabián y A. Granda (Ed), Autoevaluación de centros educativos. Cómo mejorar desde dentro (119129). Madrid: Editorial Síntesis.

Susinos, T, Calvo A. y Rojas, S. (2014). El fracaso escolar y la mejora de la escuela. Madrid: Editorial Síntesis.

Susinos, T. y Rodríguez-Hoyos, C. (2011). La educación inclusiva hoy. Reconocer al otro y crear comunidad a través del diálogo y la participación. Revista Interuniversitaria de Formación del Profesorado, 70(25), 15-30.

Villa, R.A. y Thousand, J.S. (2016). The Inclusive Education Checklist. A Self-Assessment of Best Practices. Naples: Dude Publishing.

Villa, A.; Troncoso, P. E. y Díez, F. (2015). Estructura latente y fiabilidad de las dimensiones que explican el impacto de los sistemas de gestión de calidad en los centros educativos. Revista de Investigación Educativa, 33(1), 65-82.

Fecha de entrada: 18 Marzo 2017

Fecha de revisión: 4 Marzo 2018

Fecha de aceptación: 13 Marzo 2018 